\title{
Depressive Symptoms and Perceived Doctor-Patient Communication in the Heart and Soul Study
}

\author{
Yael Schenker, $M D^{7}$, Anita Stewart, $P h D^{1,2}$, Beeya Na, $\mathrm{MPH}^{3}$, and Mary A. Whooley, $\mathrm{MD}^{1,3}$

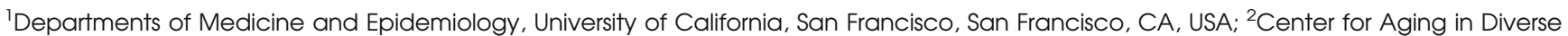 \\ Communities, Insititute for Health and Aging, University of California, San Francisco, CA, USA; ${ }^{3}$ Veterans Affairs Medical Center, San Francisco, \\ CA, USA.
}

BACKGROUND: Doctor-patient communication is an important marker of health-care quality. Little is known about the extent to which medical comorbidities, disease severity and depressive symptoms influence perceptions of doctor-patient communication in patients with chronic disease.

METHODS: In a cross-sectional study of 703 outpatients with chronic coronary disease, we evaluated the extent to which patient reports of doctor-patient communication were influenced by medical comorbidities, disease severity and depressive symptoms. We assessed patient reports of doctor-patient communication using the Explanations of Condition and Responsiveness to Patient Preferences subscales from the "Interpersonal Processes of Care" instrument. Poor doctor-patient communication was defined as a score of $<4$ (range 1 to 5 ) on either subscale. All patients completed the nine-item Patient Health Questionnaire (PHQ) for measurement of depressive symptoms and underwent an extensive evaluation of medical comorbidities and cardiac function.

RESULTS: In univariate analyses, the following patient characteristics were associated with poor reported doctor-patient communication on one or both subscales: female sex, white or Asian race and depressive symptoms. After adjusting for demographic factors, medical comorbidities and disease severity, each standard deviation (5.4-point) increase in depressive symptom score was associated with a 50\% greater odds of poor reported explanations of condition (OR 1.5, 95\% CI, 1.2-1.8; $\mathrm{p}<0.001$ ) and a $30 \%$ greater odds of poor reported responsiveness to patient preferences (OR 1.3, 95\% CI, 1.1-1.5; $\mathrm{p}=0.01)$. In contrast, objective measures of disease severity (left ventricular ejection fraction, exercise capacity, inducible ischemia) and medical comorbidities (hypertension, diabetes, myocardial infarction) were not associated with reports of doctor-patient communication.

CONCLUSIONS: In outpatients with chronic coronary heart disease, depressive symptoms are associated with perceived deficits in doctor-patient communication, while medical comorbidities and disease severity are not. These findings suggest that patient reports of

Received September 11, 2008

Revised December 2, 2008

Accepted January 26, 2009

Published online March 10, 2009 doctor-patient communication may partly reflect the psychological state of the patient.

KEY WORDS: doctor-patient communication; depression; chronic disease. J Gen Intern Med 24(5):550-6

DOI: $10.1007 / \mathrm{s} 11606-009-0937-5$

(C) Society of General Internal Medicine 2009

\section{BACKGROUND}

Doctor-patient communication is considered an important marker of health-care quality, ${ }^{1}$ and the social-psychological aspects of the patient-physician interaction are increasingly recognized as complementary to the more technical aspects of medical care. ${ }^{2,3}$ High quality doctor-patient communication involves multiple domains, including (1) building an effective relationship, (2) gathering information, (3) understanding the patient's perspective, (4) giving information and (5) decision making. ${ }^{4,5}$ Patients rate communication with their physicians as a valuable part of the medical encounter, ${ }^{6,7}$ and improved doctor-patient communication has been associated with higher patient satisfaction, ${ }^{6,8}$ self-management of chronic diseases, ${ }^{9-12}$ more appropriate prescribing of medications ${ }^{13}$ and improved health outcomes. ${ }^{14-17}$

Doctor-patient communication is particularly important in the management of chronic diseases, which may require frequent encounters with the medical system and complex treatment decisions. Patients with chronic disease often have multiple medical comorbidities and severe illness, presenting clinical challenges for physicians that may result in less attention to the social-psychological aspects of the patientphysician interaction. ${ }^{18,19}$ Patients with chronic disease also suffer disproportionately from depression, ${ }^{20,21}$ which may complicate doctor-patient communication through the challenge of managing depression along with other medical conditions or because depressed patients experience or recall communication with their physicians differently. ${ }^{22-24}$ However, little is known about the relative association of medical comorbidities, disease severity and depressive symptoms with doctor-patient communication.

We conducted this study to evaluate the association of medical comorbidities, disease severity and depressive symptoms with patient reports of doctor-patient communication in a cohort of adult patients with coronary heart disease (CHD). We chose to examine predictors of doctor-patient communication in this cohort because CHD is a chronic illness associated with high rates of medical comorbidities and depression. 


\section{METHODS}

\section{Subjects and Setting}

The Heart and Soul study is an ongoing prospective cohort study of adults with coronary heart disease (CHD). Study recruitment procedures have been published previously. ${ }^{25}$ Briefly, we used administrative databases to identify outpatients with documented CHD at two Veterans Affairs Medical Centers in Northern California (San Francisco and Palo Alto), one university medical center (University of California at San Francisco) and nine local community health clinics. We defined documented CHD as at least one of the following: a history of myocardial infarction, angiographic evidence of at least $50 \%$ stenosis in one or more coronary arteries, previous evidence of exercise-induced ischemia by treadmill or nuclear testing, a history of coronary artery revascularization or a diagnosis of CHD by an internist or a cardiologist. Subjects were excluded if they had a history of myocardial infarction in the prior 6 months, could not walk one block or were planning to move from the local area within 3 years. All participants had a primary care provider. The majority of participants spoke English; a small number of non-English-speaking patients participated through the assistance of an English-speaking family member.

A total of 1,024 subjects were enrolled between September 2000 and December 2002. After 315 participants had already been enrolled, additional funding enabled us to add instruments measuring doctor-patient communication as described below. Of the 709 remaining participants, 703 answered one or more questions about doctor-patient communication and were included in this cross-sectional study. All subjects completed a day-long initial visit that involved an extensive structured questionnaire to measure demographic variables, medical comorbidities and psychosocial functioning, a resting echocardiogram and an exercise treadmill test with stress echocardiography. The protocol was approved by the appropriate institutional review boards, and all participants provided written informed consent.

\section{Measurements}

Doctor-Patient Communication. We assessed doctor-patient communication using the two-item Explanations of Condition subscale and the four-item Responsiveness to Patient Preferences subscale from the Interpersonal Processes of Care (IPC) instrument, an established self-report questionnaire designed to measure specific components of doctor-patient communication in diverse populations. ${ }^{2}$ Details of the reliability and validity of the IPC instrument have been published previously. $^{2}$ The questionnaire was self-administered with a research assistant available in the same room to help patients understand and interpret questions as necessary. The Explanations of Condition subscale consisted of two questions: "How often did your doctor(s) give you enough information about your health problems?" and "How often did your doctor(s) make sure you understood your health problems?” The Responsiveness to Patient Preferences subscale consisted of four questions: "How often did your doctor(s) try to involve you or include you in decisions about your treatment?" "How often did your doctor(s) ask how you felt about different treatments?" How often did your doctor(s) make decisions without taking your preferences or opinions into account?" and "How often did you feel pressured by your doctor(s) to have a treatment you weren't sure you wanted?" For each question, patients were asked to report their experiences with their physician over the past 6 months on a fivepoint Likert scale ranging from "never" to "always."

We generated a summary score for each subscale by adding up the total scores within the subscale and dividing by the number of questions. For participants who did not complete all questions in the subscale $(\mathrm{N}=6$ for Explanations of Condition and N=79 for Responsiveness to Patient Preferences), we divided by the number of answered questions. Higher scores indicate better experiences of doctor-patient communication (the Likert scale was reverse scored for negative characteristics). We evaluated the IPC subscales both as continuous variables and as dichotomous variables, with a score of $<4$ on each subscale (corresponding to never/rarely/sometimes for positive characteristics and always/often/sometimes for negative characteristics) considered "poor" doctor-patient communication. ${ }^{26}$

Depressive Symptoms. We assessed depressive symptoms using the nine-item Patient Health Questionnaire (PHQ). ${ }^{27,28}$ We evaluated depressive symptoms both as a continuous variable (range of scores 0 to 27) and as an ordinal variable, using previously established categories of none-minimal (PHQ score of 0-3), mild (PHQ score of 4-9) or moderate-severe (PHQ score $\geq 10$ ) depressive symptoms. ${ }^{28}$

Medical Comorbidities and Disease Severity. Medical comorbidities (history of hypertension, diabetes and myocardial infarction) were assessed by patient questionnaire. We assessed disease severity using three objective measures of cardiac function: a resting two-dimensional echocardiogram for measurement of left ventricular ejection fraction (LVEF), an exercise treadmill test for measurement of exercise capacity and a stress echocardiogram for assessment of inducible ischemia. We performed a symptom-limited, graded exercise treadmill test according to a standard Bruce protocol, a widely adopted and validated measure of exercise capacity and ischemic heart disease. ${ }^{29}$ Peak exercise capacity was defined as the total number of metabolic equivalent tasks (METS) achieved. Poor exercise capacity was defined a priori as $<5$ METS. ${ }^{30}$ Inducible ischemia was defined as the presence of exercise-induced electrocardiographic changes or new echocardiographic wall motion abnormalities at peak exercise.

\section{Other Patient Characteristics}

Age, sex, race, education and income were measured by questionnaire. Education was measured by asking participants "What is the highest level of education that you have completed?" Participants who indicated grade 12 (or equivalent) or higher were considered to have a high school education. Income was measured by asking participants "Which of the following categories best describes your total combined household income for the past 12 months?" Primary care site was determined from administrative data.

\section{Statistical Analysis}

Our goal was to examine the associations of medical comorbidities, disease severity and depressive symptoms with patient reports of doctor-patient communication. We calculated 
internal reliability of the two IPC subscales using Cronbach's alpha. In univariate analyses, we compared differences in characteristics of participants (including demographic variables, medical comorbidities, disease severity and depressive symptoms) in patients with low (poor communication) vs. high (good communication) IPC subscale scores. Chi-squared tests were used for dichotomous variables and $t$ tests for continuous variables. We then used logistic regression to evaluate the independent association of depressive symptoms with "poor" reported doctor-patient communication, adjusted for demographic characteristics (age, sex, race, education, income), medical comorbidities (history of myocardial infarction, hypertension and diabetes) and disease severity (resting left ventricular ejection fraction, exercise capacity, inducible ischemia). These variables were hypothesized a priori to be associated with patient reports of communication.

\section{RESULTS}

Of the 703 participants, $526(75 \%)$ were male, and the mean age was 65 (range 36-97). Overall, 407 (58\%) participants were white, 126 (18\%) were black, 87 (13\%) were Asian or Pacific Islander, and 63 (9\%) were Latino. Of the 703 participants, 122 $(17 \%)$ were recruited from the San Francisco and Palo Alto VA Medical Centers, 340 (48\%) from the University of California, San Francisco, and 236 (34\%) from public health clinics in the Community Health Network of San Francisco.

Overall, 27\% (186/701) reported poor doctor-patient communication on the Explanations of Condition subscale (score $<4)$ and $41 \%(286 / 702)$ reported poor doctor-patient communication on the Responsiveness to Patient Preferences subscale (score <4). Participant responses were skewed toward reporting more positive communication experiences (Fig. 1). The Explanations of Condition subscale had a Cronbach's alpha of 0.85. The Responsiveness to Patient Preferences subscale had a Cronbach's alpha of 0.64.

Participants who reported poor explanations of condition were more likely to be female, more likely to be Asian, and less

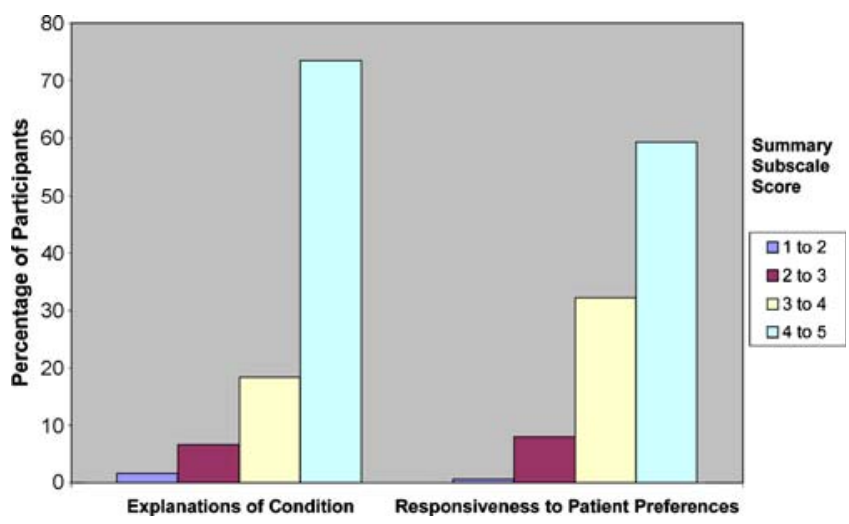

Figure 1. Distribution of summary scores on the explanations of condition and responsiveness to patient preferences subscales*. *Participants rated doctor-patient communication on a five-point Likert scale ranging from "never" to "always". A summary score was generated for each subscale by adding up the total scores within the scale and dividing by the number of answered questions. Higher scores indicate better experiences of communication. likely to be black than those who reported good explanations of condition (Table 1). Those who reported poor responsiveness to patient preferences were more likely to be white and less likely to be black than those who reported good responsiveness to patient preferences. Participants who reported poor communication on either subscale were more likely to have depressive symptoms. There were no other significant differences in the characteristics of patients reporting poor vs. good doctorpatient communication on either subscale. Ratings of communication did not differ by primary care site and were not associated with medical comorbidities or objective measures of disease severity.

Of the 703 participants, $47 \%$ had none-minimal depressive symptoms (PHQ 0-3), 32\% had mild depressive symptoms (PHQ 4-9) and 21\% had moderate-severe depressive symptoms $(\mathrm{PHQ} \geq 10)$. In describing communication with their doctor, $18 \%$ of participants with none-minimal depressive symptoms, $31 \%$ of participants with mild depressive symptoms and $39 \%$ of participants with moderate-severe depressive symptoms reported poor explanations of condition $(p<0.001$, Fig. 2). Similarly, $34 \%$ of participants with none-minimal depressive symptoms, $48 \%$ of participants with mild depressive symptoms and $45 \%$ of participants with moderate-severe depressive symptoms reported poor responsiveness to patient preferences ( $p=0.003$, Fig. 2).

In logistic regression models adjusted for age, with doctorpatient communication coded as a dichotomous outcome variable, each standard deviation (5.4 point) increase in depressive symptom score was associated with a $50 \%$ greater odds of poor reported explanations of condition (OR 1.5, 95\% CI, 1.3-1.8; p<0.0001) (Table 2) and a 30\% greater odds of poor reported responsiveness to patient preferences (OR 1.3, 95\% CI, 1.1-1.5; $\mathrm{p}=0.004$ ) (Table 3). In multivariable models, each SD increase in depressive symptoms remained strongly associated with poor reported explanations of conditions (OR $1.5,95 \%$ CI $1.2-1.8, \mathrm{p}<0.0001)$ and poor reported responsiveness to patient preferences (OR 1.3, 95\% CI 1.1-1.5, p=0.01), adjusting for age, sex, race, education, income, history of hypertension, diabetes, myocardial infarction, left ventricular ejection fraction $\leq 50 \%$, poor exercise capacity (METS $<5$ ), and inducible ischemia. We found the same association of depressive symptoms with poor doctor-patient communication in models adjusted only for age, race and sex (OR 1.5, 95\% CI 1.3-1.8; $\mathrm{p}<0.0001$ for explanations of condition; OR 1.3, 95\% CI $1.1-1.5 ; \mathrm{p}=0.006$ for responsiveness to patient preferences, data not shown). In contrast, three objective measures of disease severity (systolic function, exercise capacity and inducible ischemia) and three common medical comorbidities (history of hypertension, diabetes and myocardial infarction) were not associated with reports of doctor-patient communication on either subscale. In a sensitivity analysis including only participants who answered all questions on either subscale in the same multivariable models $(\mathrm{N}=695$ for the Explanations of Condition subscale and $\mathrm{N}=623$ for the Responsiveness to Patient Preferences subscale), results were unchanged (data not shown).

Because the association between depressive symptoms and responsiveness to patient preferences did not appear to be linear (Fig. 2), we also evaluated the association of depressive symptom score by category with reports of poor responsiveness to patient preferences (data not shown). In the multivariable model adjusted for the above-listed variables, mild depressive 
Table 1. Characteristics of Participants by Reports of Doctor-patient Communication*

\begin{tabular}{|c|c|c|c|c|c|c|}
\hline & \multicolumn{3}{|c|}{ Explanations of condition ${ }^{\dagger}$} & \multicolumn{3}{|c|}{ Responsiveness to patient preferences ${ }^{\dagger}$} \\
\hline & \multirow{2}{*}{$\frac{\text { Poor }}{(N=186)}$} & \multirow{2}{*}{$\frac{\text { Good }}{(N=515)}$} & \multirow[t]{2}{*}{$P$ value } & \multirow{2}{*}{$\frac{\text { Poor }}{(\mathrm{N}=286)}$} & \multirow{2}{*}{$\frac{\text { Good }}{(N=416)}$} & \multirow[t]{2}{*}{$P$ value } \\
\hline & & & & & & \\
\hline \multicolumn{7}{|l|}{ Demographics } \\
\hline Age, years, mean $\pm \mathrm{SD}$ & $65 \pm 11$ & $65 \pm 11$ & 0.79 & $65 \pm 11$ & $65 \pm 11$ & 0.74 \\
\hline Male & $128(69 \%)$ & 396 (77\%) & 0.03 & $211(74 \%)$ & $314(76 \%)$ & 0.61 \\
\hline Race & & & 0.02 & & & 0.01 \\
\hline White & $110(59 \%)$ & $297(58 \%)$ & & $180(63 \%)$ & $227(55 \%)$ & \\
\hline Black & $21(11 \%)$ & $105(20 \%)$ & & $34(12 \%)$ & $92(22 \%)$ & \\
\hline Asian & $31(17 \%)$ & 55 (11\%) & & $38(13 \%)$ & $48(12 \%)$ & \\
\hline Latino & $17(9 \%)$ & $46(9 \%)$ & & $25(9 \%)$ & $38(9 \%)$ & \\
\hline Other & $7(4 \%)$ & $11(2 \%)$ & & $9(3 \%)$ & $10(2 \%)$ & \\
\hline High school education & $162(88 \%)$ & 446 (87\%) & 0.78 & 254 (89\%) & 354 (85\%) & 0.1 \\
\hline Annual income $\geq 20 \mathrm{~K}$ & $102(55 \%)$ & $267(52 \%)$ & 0.56 & 139 (49\%) & $231(56 \%)$ & 0.07 \\
\hline Primary care site & & & 0.1 & & & 0.55 \\
\hline San Francisco VA & $21(11 \%)$ & $91(18 \%)$ & & $43(15 \%)$ & 69 (17\%) & \\
\hline Palo Alto VA & $1(1 \%)$ & $9(2 \%)$ & & $4(1 \%)$ & $6(1 \%)$ & \\
\hline UCSF & 99 (54\%) & $240(47 \%)$ & & $147(52 \%)$ & $192(46 \%)$ & \\
\hline Public health clinic & $64(35 \%)$ & $171(33 \%)$ & & 89 (31\%) & $147(36 \%)$ & \\
\hline \multicolumn{7}{|l|}{ Medical comorbidities } \\
\hline Hypertension & $129(70 \%)$ & 357 (69\%) & 0.92 & 194 (68\%) & 293 (70\%) & 0.5 \\
\hline Diabetes & $40(22 \%)$ & $142(28 \%)$ & 0.1 & $70(24 \%)$ & $113(27 \%)$ & 0.41 \\
\hline Myocardial infarction & 97 (52\%) & 264 (52\%) & 0.86 & $148(52 \%)$ & 213 (52\%) & 0.98 \\
\hline \multicolumn{7}{|l|}{ Disease severity } \\
\hline Resting LVEF, mean $\pm \mathrm{SD}$ & $61.2 \pm 9.6$ & $61.1 \pm 9.8$ & 0.84 & $61.6 \pm 9.4$ & $60.9 \pm 10.0$ & 0.35 \\
\hline Exercise capacity, METS & $7.5 \pm 3.3$ & $7.6 \pm 3.5$ & 0.76 & $7.6 \pm 3.4$ & $7.6 \pm 3.5$ & 0.84 \\
\hline Inducible ischemia & $33(20 \%)$ & $93(20 \%)$ & 0.98 & $49(19 \%)$ & 77 (21\%) & 0.67 \\
\hline \multicolumn{7}{|l|}{ Depressive symptoms } \\
\hline $\mathrm{PHQ}-9$, mean $\pm \mathrm{SD}$ & $7.1 \pm 5.8$ & $4.8 \pm 5.1$ & $<0.0001$ & $6.1 \pm 5.4$ & $5.0 \pm 5.3$ & 0.006 \\
\hline
\end{tabular}

Abbreviations: VA, Veterans Affairs Medical Center; UCSF, University of California, San Francisco; LVEF, left ventricular ejection fraction; METS, metabolic equivalent tasks; $P H Q-9$, nine-item patient health questionaire

*Data are given as number (percentage) except where indicated otherwise

${ }^{\dagger}$ Subscale score $<4=$ poor

symptoms (PHQ 4-9) were associated with a 60\% increased odds of poor responsiveness to patient preferences (OR 1.6, 95\% CI 1.1-2.4, p=0.01), and moderate-severe depressive symptoms $(\mathrm{PHQ} \geq 10)$ were associated with a $70 \%$ increased odds of poor responsiveness to patient preferences (OR 1.7, 95\% CI 1.1-2.8, p=0.02) compared with none-minimal depressive symptoms (PHQ 0-3).

\section{DISCUSSION}

Among patients with chronic CHD, we found that depressive symptoms were strongly associated with patient reports of poor doctor-patient communication, as measured on two subscales from the established Interpersonal Processes of Care questionnaire. Depressive symptoms were independently associated with poor reported explanations of condition and poor reported responsiveness to patient preferences, after adjustment for demographic factors, medical comorbidities and disease severity. In contrast, common medical comorbidities and objective measures of cardiac disease severity were not associated with reported doctor-patient communication. These findings raise questions about whether self-reports of doctor-patient communication are in part a reflection of the psychological state of the patient.

Several studies have explored the relationship between depression and doctor-patient communication. Patients with underlying mental disorders may be more likely to have unmet patient expectations, ${ }^{31}$ less likely to have their symptoms understood by their physicians, ${ }^{32}$ less involved in shared decision making ${ }^{33}$ and less satisfied with their care. ${ }^{34}$ To our

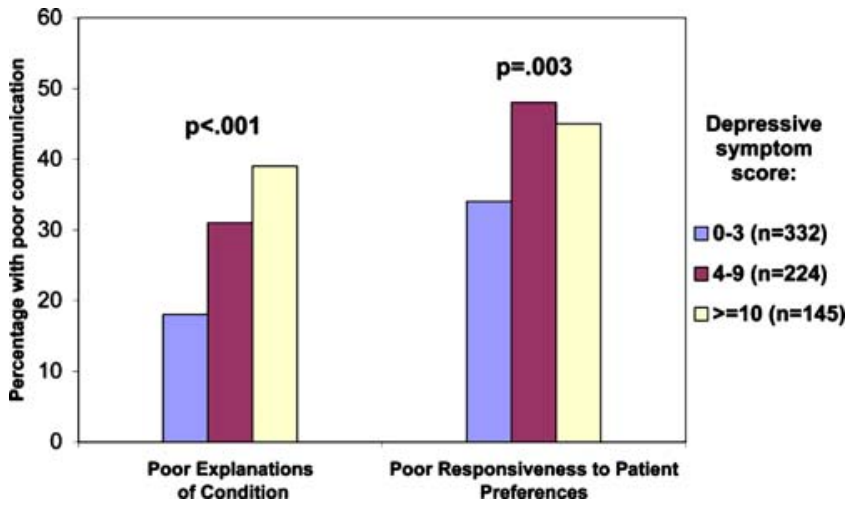

Figure 2. Percentage of participants reporting poor communication on the explanations of condition and responsiveness to patient preferences subscales, ${ }^{*}$ stratified by depressive symptom score $t$. * Subscale score <4=Poor Doctor-Patient Communication. † Depressive systems assessed using the 9-item Patient Health Questionaire (PHQ range of scores $0-27$ ). PHQ score $0-3=$ noneminimal; $\mathrm{PHQ}$ score 4-9=mild; $\mathrm{PHQ}$ score $\geq 10=$ moderate-severe. 
Table 2. Predictors of Poor Reports of Doctor-Patient Communication on the Explanations of Condition Subscale*

\begin{tabular}{|c|c|c|c|c|}
\hline & \multirow{2}{*}{$\frac{\text { Age-adjusted }}{\text { OR }(95 \% \mathrm{Cl})}$} & \multirow[b]{2}{*}{$P$ value } & \multirow{2}{*}{$\frac{\text { MV adjusted }^{\dagger}}{\text { OR }(95 \% \mathrm{Cl})}$} & \multirow[b]{2}{*}{ P value } \\
\hline & & & & \\
\hline \multicolumn{5}{|l|}{ Depressive symptoms } \\
\hline PHQ-9, per SD increase & $1.5(1.3-1.8)$ & $<0.0001$ & $1.5(1.2-1.8)$ & $<0.0001$ \\
\hline \multicolumn{5}{|l|}{ Medical comorbidities } \\
\hline History of hypertension & $1.0(0.7-1.5)$ & 0.91 & $1.1(0.7-1.7)$ & 0.63 \\
\hline History of diabetes & $0.7(0.5-1.1)$ & 0.1 & $0.7(0.4-1.1)$ & 0.09 \\
\hline History of myocardial infarction & $1.0(0.7-1.4)$ & 0.87 & $1.1(0.7-1.6)$ & 0.7 \\
\hline \multicolumn{5}{|l|}{ Disease severity } \\
\hline Systolic dysfunction (LVEF $\leq 50 \%$ ) & $1.2(0.7-1.9)$ & 0.49 & $1.4(0.8-2.6)$ & 0.23 \\
\hline Poor exercise capacity (METS $<5$ ) & $0.9(0.6-1.4)$ & 0.52 & $0.7(0.4-1.1)$ & 0.15 \\
\hline Inducible ischemia & $1.0(0.6-1.6)$ & 0.97 & $1.0(0.6-1.6)$ & 0.87 \\
\hline
\end{tabular}

Abbreviations: $P H Q-9$, nine-item patient health questionnaire; LVEF, left ventricular ejection fraction; METS, metabolic equivalents

*Poor reports of doctor-patient communication defined as Explanations of Condition Subscale score $<4$

$\dagger$ Odds ratio for a model adjusted for age, sex, race, education, income, history of hypertension, diabetes, myocardial infarction, LVEF $\leq 50 \%$, METS < 5, inducible ischemia, $\mathrm{PHQ}-9$

knowledge, only one prior study has evaluated the influence of depressive symptom severity on patient reports of doctorpatient communication across different domains. ${ }^{24}$ This study, conducted in a cohort of adults with diabetes, found that depressive symptoms were associated with worse reports of doctor-patient communication on four out of seven subscales from the same Interpersonal Processes of Care instrument. However, it was unclear how the association of depressive symptoms with poor reports of doctor-patient communication compared with medical comorbidities and objective measures of disease severity. Our study extends these important findings by reporting an association of depressive symptoms with reports of poor explanations of condition and responsiveness to patient preferences in a cohort of adults with chronic CHD and by demonstrating that the association is not explained by medical comorbidities or worse cardiac function in depressed patients.

Consistent with previous data, we found a high prevalence of depressive symptoms in patients with chronic coronary disease. $^{21,35,36}$ Depression is a significant risk factor for adverse outcomes in this population. Patients with CHD and concomitant depression are at increased risk for recurrence of cardiac events and for adverse cardiovascular outcomes after coronary artery bypass grafting surgery, ${ }^{21}$ independent of baseline cardiac disease severity. ${ }^{37}$ Depression is associated with poor health-related quality of life in patients with chronic CHD and more strongly predicts health status outcomes than objective measures of disease severity. ${ }^{25}$ Our findings suggest that patients with depression are at increased risk for experiencing poor communication with their physicians. Poor experiences of doctor-patient communication-similar to poor perceived health status-may also lead to depressive symptoms.

The lack of association between medical comorbidities or disease severity and reports of poor communication implies that medical complexity may not compromise patients' experiences of communication with their doctors to the same extent as depressive symptoms. In addition to interventions focused on clinician communication skills, efforts to improve doctorpatient communication should include increased screening and treatment of patients with depressive symptoms. Referral to mental health services, when available, may assist primary care physicians in such evaluation and treatment. Physicians may also wish to explore different strategies for communicating information and sharing decisions with patients who are depressed. Poor experiences of communication in this population may signal a need for alternative communication models.

Why patients with depressive symptoms experience poor communication is not clear. Swenson hypothesized that differences in content, process or recall among patients with depression may result in poor communication. ${ }^{24}$ Content

Table 3. Predictors of Poor Reports of Doctor-Patient Communication on the Responsiveness to Patient Preferences Subscale*

\begin{tabular}{|c|c|c|c|c|}
\hline & \multirow{2}{*}{$\frac{\text { Age-adjusted }}{\text { OR }(95 \% \mathrm{Cl})}$} & \multirow[b]{2}{*}{$P$ value } & \multirow{2}{*}{$\frac{\text { MV adjusted }^{\dagger}}{\text { OR }(95 \% \mathrm{Cl})}$} & \multirow[b]{2}{*}{$P$ value } \\
\hline & & & & \\
\hline \multicolumn{5}{|l|}{ Depressive symptoms } \\
\hline PHQ-9, per SD increase & $1.3(1.1-1.5)$ & 0.004 & $1.3(1.1-1.5)$ & 0.01 \\
\hline \multicolumn{5}{|l|}{ Medical comorbidities } \\
\hline History of hypertension & $0.9(0.6-1.2)$ & 0.5 & $1.0(0.7-1.4)$ & 0.96 \\
\hline History of diabetes & $0.9(0.6-1.2)$ & 0.43 & $1.0(0.7-1.5)$ & 0.95 \\
\hline History of myocardial infarction & $1.0(0.7-1.4)$ & 1.00 & $1.1(0.8-1.5)$ & 0.75 \\
\hline \multicolumn{5}{|l|}{ Disease severity } \\
\hline Systolic dysfunction (LVEF $\leq 50 \%$ ) & $1.0(0.6-1.6)$ & 0.96 & $1.0(0.6-1.6)$ & 0.88 \\
\hline Poor exercise capacity $(\mathrm{METS}<5)$ & $1.0(0.6-1.4)$ & 0.66 & $1.0(0.6-1.5)$ & 0.89 \\
\hline Inducible ischemia & $0.9(0.6-1.3)$ & 0.6 & $0.8(0.5-1.2)$ & 0.33 \\
\hline
\end{tabular}

Abbreviations: $P H Q-9$, nine-item patient health questionnaire; LVEF, left ventricular ejection fraction; METS, metabolic equivalents

*Poor reports of doctor-patient communication defined as Responsiveness to Patient Preferences Subscale score <4

$\dagger$ Odds ratio for a model adjusted for age, sex, race, education, income, history of hypertension, diabetes, myocardial infarction, LVEF $\leq 50 \%$, METS < 5, inducible ischemia, $P H Q-9$ 
refers to the challenge of dealing with multiple issues during a medical visit, which could lead to less time-sharing information. Our findings do not support the content explanation because communication was not associated with medical comorbidities or cardiac function. The fact that sicker patients did not report worse communication suggests that visit content did not interfere with information exchange or shared decision making. Process refers to differences in the way physicians interact with their patients. Depressed patients may have difficulty discussing their health problems with physicians, ${ }^{22}$ have a more negative affect, be less well-liked by their doctors ${ }^{38}$ and/or make physicians feel more frustrated or less engaged, ${ }^{18}$ thereby placing a strain on interpersonal relationships, which may impede physician-patient communication. Visit recall means that depressed patients may have inaccurate or skewed recollections of what happened during a visit. Depressed patients may report poor communication in part because they view everything in a negative light. Alternatively, difficulty with concentration, a symptom of major depression, ${ }^{39}$ may impair patient recall of communication during the medical visit.

While the process of communication may be objectively worse in patients with depressive symptoms, it is also possible that patients with depression perceive the same interactions differently. Depressive symptoms are associated with poor reports of doctor-patient communication, and the perceived quality of doctor-patient communication predicts patient satisfaction. ${ }^{40}$ Thus, if patients with depressive symptoms report lower satisfaction with their physicians, is this because depressed patients perceive things differently or because their physicians are actually providing poorer quality care? The possibility that patients with depressive symptoms view clinical encounters through different lenses raises questions about the validity of patient self-report questionnaires used as measures of health-care quality.

Several limitations must be considered in interpreting our results. First, we evaluated two aspects of doctor-patient communication, explanations of condition and responsiveness to patient preferences, using two subscales from the Interpersonal Processes of Care instrument. Our study did not address other important components of doctor-patient communication including empathy, respectfulness, listening or empowerment. ${ }^{2,3,41}$ While explanations of condition and responsiveness to patient preferences are critical aspects of care, it is possible that the inclusion of additional measures of doctor-patient communication would have provided different results. Second, we studied patients with coronary heart disease, so our results may not generalize to patients with other illnesses. However, CHD is a common chronic illness requiring frequent physician visits and associated with multiple medical comorbidities and high rates of depression. Third, it is possible that depression itself may have presented a barrier to participation in this study. However, we found a high prevalence of depressive symptoms consistent with previous data. ${ }^{36}$ Fourth, our participants spoke English, and the majority were male and white. Although the Interpersonal Processes of Care instrument was designed to compare doctorpatient communication across diverse racial/ethnic groups, our study sample did not allow us to make such comparisons. Fifth, we did not collect information about individual physicians. While previous work has demonstrated an association between physician characteristics and patient reports of doctor-patient communication, ${ }^{42}$ we were unable to evaluate physician predictors of communication or account for clustering at the physician level. Sixth, the Interpersonal Processes of Care instrument is a patient self-report questionnaire; we did not observe doctor-patient communication directly. While patient perceptions of communication are important in and of themselves, direct observation might have allowed us to examine whether perceived differences in communication were more strongly associated with visit process or recall. Finally, this was a cross-sectional study; thus, we were unable to evaluate causality.

\section{CONCLUSION}

In summary, patients with depressive symptoms are more likely to report poor doctor-patient communication than those without depressive symptoms. Whether differences in perceived communication are due to differences in the communication process or to differences in recall among patients with depressive symptoms deserves further study. Patient reports are important in and of themselves and signal a need for increased awareness of the influence of depressive symptoms on experiences of doctor-patient communication. However, further correlation with physician reports and/or direct observation may be necessary before such reports can be used as objective measures of health-care quality.

Acknowledgements: This study was supported by the Department of Veterans Epidemiology Merit Review Program; the Department of Veterans Affairs Health Services Research and Development service; the National Heart Lung and Blood Institute (RO1 HLO79235); the American Federation for Aging Research (Paul Beeson Scholars Program); the Robert Wood Johnson Foundation (Generalist Physician Faculty Scholars Program); the Ischemia Research and Education Foundation; and the Department of Health and Human Services Health Resources and Services Administration (DHHS HRSA D55HP05165). Findings were presented at the Society of General Internal Medicine National Meeting on April 11, 2008.

\section{Conflicts of Interest: None disclosed.}

Corresponding Author: Yael Schenker, MD; Departments of Medicine and Epidemiology, University of California, San Francisco, Box 1364, San Francisco, CA 94143, USA (e-mail: yael.schenker@ ucsf.edu).

\section{REFERENCES}

1. Institute of Medicine. Crossing the Quality Chasm. A New Health System for the 21 st Century. Institute of Medicine: Washington, D.C.; 2001

2. Stewart AL, Napoles-Springer A, Perez-Stable EJ. Interpersonal processes of care in diverse populations. Milbank Q. 1999;77(3):305-39.

3. Stewart AL, Napoles-Springer AM, Gregorich SE, Santoyo-Olsson J. Interpersonal processes of care survey: patient-reported measures for diverse groups. Health Serv Res. 2007;42:1235-56.

4. Cole SA, Bird J. The medical interview: The three-function approach. 2nd ed. St Louis: Mosby; 2000.

5. Makoul G. Essential elements of communication in medical encounters: the Kalamazoo consensus statement. Acad Med. 2001;76(4): 390-3.

6. Fung CH, Elliott MN, Hays RD, et al. Patients' preferences for technical versus interpersonal quality when selecting a primary care physician. Health Serv Res. 2005;40:957-77.

7. Laine C, Davidoff F, Lewis CE, et al. Important elements of outpatient care: a comparison of patients' and physicians' opinions. Ann Intern Med. 1996;125:640-5. 
8. Golin C, DiMatteo MR, Duan N, Leake B, Gelberg L. Impoverished diabetic patients whose doctors facilitate their participation in medical decision making are more satisfied with their care. J Gen Intern Med. 2002;17:857-66

9. Bultman DC, Svarstad BL. Effects of physician communication style on client medication beliefs and adherence with antidepressant treatment. Patient Educ Couns. 2000;40:173-85.

10. Heisler M, Bouknight RR, Hayward RA, Smith DM, Kerr EA. The relative importance of physician communication, participatory decision making, and patient understanding in diabetes self-management. J Gen Intern Med. 2002;17:243-52.

11. Piette JD, Schillinger D, Potter MB, Heisler M. Dimensions of patientprovider communication and diabetes self-care in an ethnically diverse population. J Gen Intern Med. 2003;18:624-33.

12. Schneider J, Kaplan SH, Greenfield S, Li W, Wilson IB. Better physician-patient relationships are associated with higher reported adherence to antiretroviral therapy in patients with HIV infection. J Gen Intern Med. 2004;19:1096-103.

13. Epstein RM, Shields CG, Franks P, Meldrum SC, Feldman M, Kravitz RL. Exploring and validating patient concerns: relation to prescribing for depression. Ann Fam Med. 2007;5:21-8.

14. Greenfield S, Kaplan S, Ware JE Jr. Expanding patient involvement in care. Effects on patient outcomes. Ann Intern Med. 1985;102: 520-8.

15. Greenfield S, Kaplan SH, Ware JE Jr, Yano EM, Frank HJ. Patients participation in medical care: effects on blood sugar control and quality of life in diabetes. J Gen Intern Med. 1988;3:448-57.

16. Kaplan SH, Greenfield S, Ware JE Jr. Assessing the effects of physician-patient interactions on the outcomes of chronic disease. Med Care. 1989;27:S110-27.

17. Schillinger D, Piette J, Grumbach K, et al. Closing the loop: physician communication with diabetic patients who have low health literacy. Arch Intern Med. 2003;163:83-90.

18. Hall JA, Roter DL, Milburn MA, Daltroy LH. Patients' health as a predictor of physician and patient behavior in medical visits. A synthesis of four studies. Med Care. 1996;34:1205-18.

19. Hall JA, Milburn MA, Roter DL, Daltroy LH. Why are sicker patients less satisfied with their medical care? Tests of two explanatory models. Health Psychol. 1998;17:70-5.

20. Egede LE. Major depression in individuals with chronic medical disorders: prevalence, correlates and association with health resource utilization, lost productivity and functional disability. Gen Hosp Psychiatry. 2007;29:409-16.

21. Whooley MA. Depression and cardiovascular disease: healing the broken-hearted. JAMA. 2006;295:2874-81.

22. Gask L, Rogers A, Oliver D, May C, Roland M. Qualitative study of patients' perceptions of the quality of care for depression in general practice. Br J Gen Pract. 2003;53:278-83.

23. Jackson JL, Kroenke K. Difficult patient encounters in the ambulatory clinic: clinical predictors and outcomes. Arch Intern Med. 1999;159:106975.

24. Swenson SL, Rose M, Vittinghoff E, Stewart A, Schillinger D. The influence of depressive symptoms on clinician-patient communica- tion among patients with type 2 diabetes. Med Care. 2008;46:25765.

25. Ruo B, Rumsfeld JS, Hlatky MA, Liu H, Browner WS, Whooley MA. Depressive symptoms and health-related quality of life: the Heart and Soul Study. JAMA. 2003;290:215-21.

26. Schillinger D, Bindman A, Wang F, Stewart A, Piette J. Functiona health literacy and the quality of physician-patient communication among diabetes patients. Patient Educ Couns. 2004;52:315-23.

27. Spitzer RL, Kroenke K, Williams JB. Validation and utility of a selfreport version of PRIME-MD: the PHQ primary care study. Primary Care Evaluation of Mental Disorders. Patient Health Questionnaire. JAMA. 1999;282:1737-44

28. Kroenke K, Spitzer RL, Williams JB. The PHQ-9: validity of a brief depression severity measure. J Gen Intern Med. 2001;16:606-13.

29. Hill J, Timmis A. Exercise tolerance testing. BMJ. 2002;324 (7345):1084-7.

30. Myers J, Prakash M, Froelicher V, Do D, Partington S, Atwood JE. Exercise capacity and mortality among men referred for exercise testing. N Engl J Med. 2002;346:793-801.

31. Jackson JL, Kroenke $\mathbf{K}$. The effect of unmet expectations among adults presenting with physical symptoms. Ann Intern Med. 2001;134(9 Pt 2):889-97.

32. Zastrow A, Faude V, Seyboth F, Niehoff D, Herzog W, Lowe B. Risk factors of symptom underestimation by physicians. J Psychosom Res. 2008;64(5):543-51.

33. Young HN, Bell RA, Epstein RM, Feldman MD, Kravitz RL. Physicians' shared decision-making behaviors in depression care. Arch Intern Med. 2008;168(13): 1404-8.

34. Jackson JL, Chamberlin J, Kroenke K. Predictors of patient satisfaction. Soc Sci Med. 2001;52(4):609-20.

35. Evans DL, Charney DS, Lewis L, et al. Mood disorders in the medically ill: scientific review and recommendations. Biol Psychiatry. 2005;58 (3): 175-89.

36. Moussavi S, Chatterji S, Verdes E, Tandon A, Patel V, Ustun B.s Depression, chronic diseases, and decrements in health: results from the World Health Surveys. Lancet. 2007;370(9590):851-8.

37. Lett $\mathbf{H}$, Ali S, Whooley $\mathbf{M}$. Depression and cardiac function in patients with stable coronary heart disease: findings from the Heart and Sou study. Psychosom Med. 2008;70(4):444-9.

38. Hall JA, Horgan TG, Stein TS, Roter DL. Liking in the physicianpatient relationship. Patient Educ Couns. 2002;48:69-77.

39. Diagnostic and Statistical Manual of Mental Disorders, Fourth Edition Washington, DC: American Psychiatric Association; 1994

40. Kim SC, Kim S, Boren D. The quality of therapeutic alliance between patient and provider predicts general satisfaction. Mil Med. 2008;173:8590.

41. Lein C, Wills CE. Using patient-centered interviewing skills to manage complex patient encounters in primary care. J Am Acad Nurse Pract. 2007; 19(5):215-20.

42. Fernandez A, Schillinger D, Grumback K, et al. Physician language ability and cultural competence. An exploratory study of communication with Spanish-speaking patients. J Gen Intern Med. 2004;19 (2): 167-74 